\title{
Features of hemodynamic and metabolic disorders in obese patients with resistant hypertension
}

\author{
Anna Shalimova ${ }^{1,2}$, Valentyna Psarova ${ }^{3}$, Maryna Kochuieva4, Olena Kolesnikova', Anna Isayeva1, \\ Vira Zlatkina', Valeriya Nemtsova ${ }^{2}$ \\ 'Government Institution "L.T. Malaya Therapy National Institute of the National Academy of Medical Sciences of Ukraine", Kharkiv, Ukraine \\ ${ }^{2}$ Kharkiv National Medical University, Kharkiv, Ukraine \\ ${ }^{3}$ Sumy State University, Sumy, Ukraine \\ ${ }^{4}$ Kharkiv Medical Academy of Postgraduate Education, Kharkiv, Ukraine
}

\begin{abstract}
Background. The aim was to establish the features of hemodynamic and metabolic parameters in obese patients with true and pseudo-resistant arterial hypertension (AH).

Material and methods. The study included 200 patients with uncontrolled $\mathrm{AH}$ and obesity. Patients were initially prescribed dual antihypertensive therapy. Those patients who did not reach target blood pressure (BP) levels after 3 months on dual therapy were additionally prescribed a third antihypertensive drug. Of the 98 patients who were assigned to triple therapy, 48 patients did not reach target BP (27 patients had pseudo-resistant and 21 patients had true resistant $\mathrm{AH}$ ). These patients were additionally prescribed a fourth antihypertensive drug (spironolactone). The effectiveness of the treatment was evaluated 6 months after the start of antihypertensive therapy.

Results. After 6 months of therapy, unlike patients without resistance, individuals with resistant AH had more pronounced cardiovascular remodeling and metabolic disorders, disbalance of oxidative stress-antioxidant protection, proinflammatory activity and higher activity of the renin-angiotensin-aldosterone system. Patients with true resistance differed from pseudo-resistant patients by having significantly lower body mass index (BMI); in the absence of differences in BP levels, cardiovascular remodeling, lipid and carbohydrate profiles, patients with true resistance had significantly higher levels of aldosterone, higher activity of oxidative stress system, lower levels of general antioxidant protection, higher adiponectin levels, and lower leptin level.

Conclusions. Obese patients with true resistance differed from pseudo-resistant patients by having significantly lower BMI, higher aldosterone levels, more pronounced imbalance of the system of oxidative stress-antioxidant protection and less pronounced adipokine imbalance.
\end{abstract}

Key words: resistant hypertension; obesity; hemodynamic disorders; metabolic disorders

Arterial Hypertens. 2020, vol. 24, no. 1, pages: 22-29

DOI: $10.5603 /$ AH.a2020.0002

Address for correspondence: Anna Shalimova

Government Institution L.T. Malaya Therapy National Institute of the National Academy of Medical Sciences of Ukraine, Malaya 2a av.

61039 Kharkiv, Ukraine; e-mail: anna.shalimova83@gmail.com 


\section{Introduction}

Resistant hypertension (RH) is an important cause of cardiovascular morbidity and mortality. According to the definition of resistance presented in the 2018 ESC/ESH Guidelines for the management of arterial hypertension (AH), RH should be diagnosed when the recommended treatment strategy fails to lower office systolic and diastolic blood pressure (SBP and DBP) values to less than $140 \mathrm{~mm} \mathrm{Hg}$ and/or less than 90 $\mathrm{mm} \mathrm{Hg}$, respectively, and the inadequate control of BP is confirmed by ambulatory or home BP monitoring in patients whose adherence to therapy has been confirmed [1]. As indicated in the specified Guidelines, the treatment strategy should include appropriate lifestyle measures and treatment with optimal or best tolerated doses of three or more drugs, which should include a diuretic, typically an angiotensin-converting enzyme (ACE) inhibitor (or an angiotensin II receptor blockers) and a calcium channel blockers (CCB) [1].

At the same time, pseudo-resistant hypertension (due to low compliance with treatment, the whitecoat phenomenon, increased calcification of the shoulder arteries and a number of other causes) and true resistance should be distinguished. Both true and pseudo-resistant hypertensions represent a big problem not only in BP control, but also in those possible adverse cardiovascular events, the development of which is associated with failure to achieve target BP levels.

Resistant hypertension patients require a multimodal approach to control BP. In most patients, $\mathrm{RH}$ can be influenced by optimizing combination antihypertensive therapy to improve compliance and lifestyle correction [1,2]. As indicated in the 2018 ESC/ESH Guidelines [1], in the case of $\mathrm{RH}$, an antagonist of mineralocorticoid receptors should be additionally prescribed to patients, due to its effect on a number of pathogenetic mechanisms such as the suppression of macrophage infiltration and improvement of endothelium-dependent vasodilatation (EDVD) [3-6]. There is evidence of a more pronounced hypotensive effect of aldosterone blockers in patients with increased waist circumference than in patients with its normal values [7-9].

Given the high prevalence of comorbidity of $\mathrm{AH}$ and obesity on the one hand and a large percentage of people with insufficiently controlled $\mathrm{AH}$ on the other hand, the study of the features of true resistance and pseudo-resistance in obese patients deserves attention.

The aim of the study was to establish the features of hemodynamic and metabolic parameters in obese patients with true and pseudo-resistant $\mathrm{AH}$.

\section{Material and methods}

The study included 200 patients with uncontrolled $\mathrm{AH}$ and obesity classes I-II (the age $45-55$ years). Antihypertensive therapy was prescribed in accordance with the 2018 ESC/ESH Guidelines for the management of AH [1]. Dual antihypertensive therapy (ACE inhibitor and CCB) was administered to patients first. Those patients who did not reach the target BP levels of dual therapy after 3 months were further prescribed a third antihypertensive drug (the thiazide-like diuretic indapamide). Of the 98 patients who were assigned to triple therapy, 48 patients did not reach the target BP levels: 27 patients had pseudo-resistant hypertension (due to low compliance with treatment and insufficient adherence to the physician's instructions to ensure adequate levels of physical activity), and 21 patients had true $\mathrm{RH}$ (did not achieve target BP levels with triple antihypertensive therapy and sufficient physical activity). These patients were additionally prescribed a fourth antihypertensive drug - spironolactone. The effectiveness of the treatment was evaluated 6 months after the start of antihypertensive therapy.

Adherence to treatment was evaluated on the basis of the patient's diary, which included the time of taking a fixed combination of antihypertensive drugs and BP levels in the morning and evening hours. In addition, in the diary, the patient entered the information on the number of steps taken during the day, which were measured using a pedometer.

Physical examination of patients included measurements of height, body weight, and BMI calculation. Patients' waist circumference (WC) and hip circumference (HC) were measured, and waist-tohip ratio was calculated. Office BP was measured in accordance with the 2018 ESC/ESH Guidelines for the management of $\mathrm{AH}$ [1]. Pulse BP was evaluated as the difference between SBP and DBP. Average BP was calculated by the formula:

$$
\text { Average } B P=0.42 \times(S B P-D B P)+D B P
$$

Lipid metabolism was assessed by total cholesterol, triglycerides, LDL and HDL cholesterol. Carbohydrate metabolism was assessed by blood glucose, $\mathrm{HbA1c}$ and insulin levels. Insulin resistance (IR) was determined by the HOMA model. The activity of the renin-angiotensin-aldosterone system (RAAS) was evaluated by the levels of aldosterone and renin plas$\mathrm{ma}$, as well as by the aldosterone-renin ratio (ARR). The inflammatory activity was evaluated by the levels of interleukin 6 (IL-6) and C-reactive protein (CRP). The functional state of adipose tissue was assessed by 
blood levels of leptin and adiponectin. The state of the prooxidant system was evaluated by the levels of molecular products of lipid peroxidation - diene conjugates (DC) and malonic dialdehyde (MDA), and the state of the antioxidant protection system - by the total antioxidant activity.

Morphofunctional properties of the myocardium were evaluated during ultrasound examination of the heart in one-dimensional, two-dimensional and Doppler modes by conventional methods. The volumes of left and right atria (LA and RA, respectively), end-systolic and end-diastolic diameters (ESD and EDD, respectively) of the left ventricle (LV), diameters of LA and aorta (LAD and AD, respectively) were evaluated. The ejection fraction (EF) was calculated by the formula:

$$
E F=(E D V-E S V) / E D V
$$

where ESV and EDV are the end-systolic and enddiastolic LV volumes, respectively.

The thickness of the posterior wall of the LV and the thickness of the interventricular septum in the systole (TPWs and TIVSs, respectively) and diastole (TPWd and TIVSd, respectively) were measured. The relative wall thickness of the LV (RWT) was calculated by the formula:

$$
R W T=(T P W d+T I V S d) / E D D
$$

The LV myocardial mass index (MMILV) was calculated as the ratio of the LV myocardial mass (MMLV) to the surface area of the body (S):

$$
M M I L V=M M L V / S
$$

Left ventricular diastolic function was evaluated by pulmonary artery blood flow and transmitral diastolic blood flow in pulsed Doppler with the determination of the following parameters: maximum early LV filling rate in spectral mode (E), maximum late (atrial) filling speed (A), ratio of maximal rates of early and late filling of LV at spectral mode (E/A), time of isovolumic relaxation of LV (IVRT), time of deceleration early diastolic flow rate (DT), maximum early LV filling rate at tissue mode (e), mean pulmonary artery pressure (AP) by Kitabatake, ratio of $E$ and $e(E / e)$.

The degree of EDVD was determined in the sample with reactive hyperemia. At the same time, the intima-media thickness (IMT) in the carotid artery (CA) was measured $2 \mathrm{~cm}$ more proximal to the bifurcation of the common CA. The pulse wave velocity (PWV) in the CA was determined by the W-Track method; determination of the PWV in the abdominal aorta (AA) was performed using a phased sensor.

The statistical processing of the obtained data was carried out using the package of statistical software "SPSS 17" (IBM), Microsoft Office Exel-2003. The data are presented as mean values \pm standard deviation. Significance was set at a $\mathrm{p}$ value of $<0.05$ in all cases.

\section{Results}

The comparative assessment of patients' values in the presence and absence of $\mathrm{RH}$ after 6 months of therapy was first of all performed (Tab. 1). It was found that after 6 months of therapy, patients with $\mathrm{RH}$ had higher BMI and higher BP levels compared to patients without this condition.

It should be noted that patients with RH also had more pronounced cardiovascular remodeling (higher IMT CA, IVS, TPW, TIVS LVM, E/e and lower $\mathrm{EF}$ ), higher levels of triglycerides, insulin, HbA1c, more pronounced IR (which was confirmed by greater HOMA-IR), greater imbalance of adipokines, proinflammatory activity and higher RAAS activity (Tab. 1).

A comparative assessment of pseudo-resistance and true resistance showed that patients with true resistance differed from pseudo-resistant patients by having significantly lower BMI $(\mathrm{p}=0.02)($ Tab. 2$)$. In addition, in the absence of differences in BP levels, cardiovascular remodeling, and lipid and carbohydrate profiles, patients with true resistance had significantly higher levels of aldosterone $(p=0.04)$, higher activity of oxidative stress (MDA, $\mathrm{p}=0.01$ and DC, $p=0.03)$, a lower level of total antioxidant protection $(p=0.00)$, a higher level of adiponectin $(p=0.00)$, and a lower level of leptin $(p=0.00)$, compared with pseudo-resistant patients.

\section{Discussion}

The literature suggests that between $50 \%$ and $80 \%$ of patients with $\mathrm{AH}$ who are prescribed antihypertensive drugs show suboptimal adherence to treatment. In many cases, this high percentage of failure to reach target BP levels is at least in part due to the large number of drugs, the complexity of their dosage, the high incidence of adverse reactions, poor patientphysician relationships, and the inertia of the clinician with reduced adherence to treatment $[2,10]$.

Renin-angiotensin-aldosterone system plays a key role in the BP regulation, and its excessive activity 
Table 1. Comparative assessment of indicators in patients 6 months after treatment, depending on the presence of resistant arterial hypertension $(\mathrm{AH})$

\begin{tabular}{|c|c|c|c|}
\hline Indicators & $\begin{array}{l}\text { Resistant AH is absent } \\
\quad(n=152)\end{array}$ & $\begin{array}{l}\text { Resistant AH is present } \\
\qquad(\mathrm{n}=48)\end{array}$ & $\mathbf{p}$ \\
\hline Weight [kg] & $92.62 \pm 9.65$ & $98.56 \pm 7.51$ & 0.00 \\
\hline $\mathrm{BMI}\left[\mathrm{kg} / \mathrm{m}^{2}\right]$ & $31.85 \pm 2.53$ & $34.44 \pm 1.37$ & 0.00 \\
\hline Waist [cm] & $99.13 \pm 6.79$ & $101.65 \pm 6.35$ & 0.02 \\
\hline Hip [cm] & $108.38 \pm 10.39$ & $105.52 \pm 5.54$ & 0.07 \\
\hline Waist-to-hip ratio & $0.96 \pm 0.60$ & $0.97 \pm 0.08$ & 0.97 \\
\hline SBP $[\mathrm{mm} \mathrm{Hg}]$ & $136.34 \pm 1.83$ & $138.05 \pm 1.43$ & 0.00 \\
\hline $\mathrm{DBP}[\mathrm{mm} \mathrm{Hg}]$ & $86.43 \pm 1.01$ & $88.23 \pm 0.75$ & 0.00 \\
\hline Pulse BP [mm Hg] & $48.90 \pm 2.03$ & $49.13 \pm 1.62$ & 0.49 \\
\hline Average BP [mm Hg] & $103.87 \pm 0.88$ & $105.86 \pm 0.92$ & 0.00 \\
\hline Heart rate [bpm] & $68.89 \pm 3.22$ & $68.83 \pm 3.70$ & 0.92 \\
\hline IMT CA [mm] & $0.87 \pm 0.08$ & $0.93 \pm 0.08$ & 0.00 \\
\hline IMT CA bifurcation [mm] & $1.26 \pm 0.15$ & $1.29 \pm 0.13$ & 0.19 \\
\hline PWV CA [m/s] & $7.84 \pm 1.04$ & $7.82 \pm 0.99$ & 0.90 \\
\hline PWV AA [m/s] & $7.75 \pm 1.03$ & $7.80 \pm 1.00$ & 0.75 \\
\hline EDVD (\%) & $7.89 \pm 1.29$ & $7.92 \pm 0.91$ & 0.86 \\
\hline Total cholesterol [mmol/L] & $5.72 \pm 0.47$ & $5.79 \pm 0.38$ & 0.31 \\
\hline Triglycerides [mmol/L] & $1.70 \pm 0.28$ & $1.95 \pm 0.42$ & 0.00 \\
\hline LDL cholesterol [mmol/L] & $4.56 \pm 0.47$ & $4.72 \pm 0.45$ & 0.08 \\
\hline HDL cholesterol, [mmol/L] & $1.04 \pm 0.13$ & $1.03 \pm 0.19$ & 0.37 \\
\hline Glucose $[\mathrm{mmol} / \mathrm{L}]$ & $4.66 \pm 0.30$ & $4.69 \pm 0.29$ & 0.49 \\
\hline Insulin $[\mu \mathrm{U} / \mathrm{mL}]$ & $11.19 \pm 4.02$ & $16.36 \pm 2.80$ & 0.00 \\
\hline HOMA-IR & $2.32 \pm 0.85$ & $3.39 \pm 0.60$ & 0.00 \\
\hline $\mathrm{HbA}_{1 \mathrm{c}}(\%)$ & $4.68 \pm 0.30$ & $4.82 \pm 0.32$ & 0.02 \\
\hline Total antioxidant activity [mmol/L] & $1.26 \pm 0.06$ & $1.18 \pm 0.06$ & 0.00 \\
\hline $\mathrm{MDA}[\mathrm{nmol} / \mathrm{mL}]$ & $30.38 \pm 2.53$ & $32.80 \pm 1.72$ & 0.00 \\
\hline $\mathrm{DC}[\mathrm{nmol} / \mathrm{mL}]$ & $27.09 \pm 3.57$ & $31.08 \pm 3.45$ & 0.00 \\
\hline IL-6 [ng/mL] & $106.75 \pm 6.94$ & $109.82 \pm 7.53$ & 0.01 \\
\hline $\mathrm{CRP}[\mathrm{ng} / \mathrm{mL}]$ & $4.26 \pm 0.75$ & $4.51 \pm 0.72$ & 0.04 \\
\hline Aldosteron [ng/dl] & $14.27 \pm 1.62$ & $14.76 \pm 1.32$ & 0.32 \\
\hline Renin, ng/ml/hour & $2.19 \pm 0.46$ & $2.53 \pm 0.47$ & 0.01 \\
\hline ARR & $7.60 \pm 1.56$ & $6.11 \pm 1.54$ & 0.00 \\
\hline Adiponectin [ng/mL] & $7.49 \pm 1.06$ & $8.09 \pm 0.98$ & 0.00 \\
\hline Leptin [ng/mL] & $11.61 \pm 2.20$ & $12.73 \pm 2.03$ & 0.00 \\
\hline IVSd [sm] & $1.10 \pm 0.12$ & $1.15 \pm 0.11$ & 0.01 \\
\hline IVSs [sm] & $1.30 \pm 0.14$ & $1.36 \pm 0.13$ & 0.01 \\
\hline TPWd [sm] & $1.09 \pm 0.13$ & $1.14 \pm 0.14$ & 0.02 \\
\hline TPWs [sm] & $1.51 \pm 0.33$ & $1.55 \pm 0.36$ & 0.53 \\
\hline TIVSd [sm] & $4.98 \pm 0.32$ & $5.04 \pm 0.41$ & 0.30 \\
\hline TIVSs [sm] & $3.12 \pm 0.25$ & $3.20 \pm 0.30$ & 0.04 \\
\hline $\mathrm{EF}(\%)$ & $67.13 \pm 3.20$ & $65.86 \pm 2.24$ & 0.01 \\
\hline LVM [g] & $244.72 \pm 60.55$ & $267.79 \pm 73.88$ & 0.03 \\
\hline LVMMI $\left[\mathrm{g} / \mathrm{m}^{2}\right]$ & $120.00 \pm 29.06$ & $128.69 \pm 35.85$ & 0.09 \\
\hline
\end{tabular}


Table 1. Comparative assessment of indicators in patients 6 months after treatment, depending on the presence of resistant arterial hypertension (AH)

\begin{tabular}{|l|c|c|c|}
\hline Indicators & $\begin{array}{c}\text { Resistant AH is absent } \\
(\mathbf{n}=\mathbf{1 5 2})\end{array}$ & $\begin{array}{c}\text { Resistant AH is present } \\
\text { (n= 48) }\end{array}$ & $\mathbf{p}$ \\
\hline RWT & $0.44 \pm 0.04$ & $0.46 \pm 0.04$ & 0.01 \\
\hline LAD $[\mathrm{mm}]$ & $37.33 \pm 3.03$ & $37.29 \pm 3.54$ & 0.95 \\
\hline AD $[\mathrm{mm}]$ & $32.47 \pm 1.60$ & $32.07 \pm 0.59$ & 0.09 \\
\hline Mean pulmonary AP $[\mathrm{mm} \mathrm{Hg}]$ & $15.54 \pm 3.12$ & $15.71 \pm 2.97$ & 0.75 \\
\hline RAV [mL] & $38.31 \pm 4.90$ & $37.40 \pm 3.81$ & 0.24 \\
\hline LAV [mL] & $46.71 \pm 4.64$ & $48.31 \pm 4.09$ & 0.03 \\
\hline E $[\mathrm{cm} / \mathrm{s}]$ & $69.91 \pm 11.68$ & $67.49 \pm 6.18$ & 0.17 \\
\hline A [cm/s] & $78.99 \pm 11.11$ & $77.67 \pm 8.05$ & 0.46 \\
\hline E/A & $0.94 \pm 0.19$ & $0.90 \pm 0.09$ & 0.19 \\
\hline DT $[\mathrm{s}]$ & $0.15 \pm 0.09$ & $0.15 \pm 0.09$ & 0.96 \\
\hline IVRT $[\mathrm{s}]$ & $0.10 \pm 0.02$ & $0.11 \pm 0.02$ & 0.11 \\
\hline E $[\mathrm{cm} / \mathrm{s}]$ & $12.61 \pm 2.54$ & $11.57 \pm 2.28$ & 0.01 \\
\hline E/e & $5.67 \pm 1.04$ & $6.04 \pm 1.25$ & 0.04 \\
\hline
\end{tabular}

A — maximum late (atrial) filling speed; AA — aortic arch; AP — artery pressure; ARR — aldosterone-renin ratio; BMI — body mass index; BP — blood pressure; CA — carotid artery; CRP — C-reactive protein; $\mathrm{DBP}$ - diastolic blood pressure; $\mathrm{DC}$ - diene conjugates; $\mathrm{DT}$ - time of deceleration early diastolic flow rate; $\mathrm{E}$ - filling rate in spectral mode; $\mathrm{e}-$ maximum early LV filling rate at tissue mode; $\mathrm{E} / \mathrm{A}$ - ratio of maxima rates of early and late filling of $L V$ at spectral mode; $\mathrm{E} / \mathrm{e}$ - ratio of $\mathrm{E}$ and e; $\mathrm{EDVD}$ - endothelium-dependent vasodilatation; $\mathrm{EF}$ — ejection fraction; $\mathrm{HbA} \mathrm{I}_{\mathrm{lc}}$ - glycated hemoglobin; $\mathrm{HDL}$ - high density lipoprotein; IL-6 — interleukin 6; HOMA-IR — Homeostatic Model Assessment for Insulin Resistance; IMT — intima-media thickness; IVRT — time of isovolumic relaxation of LV; IVSd, interventricular septum (diastole): IVSs, interventricular septum (systole); LDL — low-density lipoprotein; LVM — left ventricular mass; MDA — malonic dialdehyde; RAV — right atrial volume; LAV — left atrial volume; SBP — systolic blood pressure; PWV — pulse wave velocity; TIVSd - thickness of the interventricular septum (diastole); TIVSs - thickness of the interventricular septum (systole); TPWd — thickness of the posterior wall of the left ventricle in diastole; TIVSd — the thickness of the interventricular septum in diastole

Table 2. Comparison of patients with pseudo-resistant and true resistant arterial hypertension (AH)

\begin{tabular}{|c|c|c|c|}
\hline Indicators & $\begin{array}{l}\text { Pseudo-resistant AH } \\
\text { (n=27) }\end{array}$ & $\begin{array}{l}\text { True resistant AH } \\
\quad(\mathrm{n}=21)\end{array}$ & $\mathbf{p}$ \\
\hline Weight [kg] & $99.56 \pm 8.37$ & $97.29 \pm 6.21$ & 0.43 \\
\hline BMI $\left[\mathrm{kg} / \mathrm{m}^{2}\right]$ & $34.83 \pm 1.28$ & $33.93 \pm 1.35$ & 0.02 \\
\hline Waist [cm] & $101.56 \pm 6.73$ & $101.76 \pm 6.00$ & 0.91 \\
\hline Hip [cm] & $105.26 \pm 5.40$ & $105.86 \pm 5.82$ & 0.79 \\
\hline Waist-to-hip ratio & $0.97 \pm 0.08$ & $0.96 \pm 0.08$ & 0.95 \\
\hline $\mathrm{SBP}[\mathrm{mm} \mathrm{Hg}]$ & $137.30 \pm 1.52$ & $137.43 \pm 1.48$ & 0.71 \\
\hline $\mathrm{DBP}[\mathrm{mm} \mathrm{Hg}]$ & $88.25 \pm 0.82$ & $88.41 \pm 0.84$ & 0.93 \\
\hline Pulse BP [mm Hg] & $49.07 \pm 1.62$ & $49.19 \pm 1.66$ & 0.76 \\
\hline Average BP [mm Hg] & $104.03 \pm 0.96$ & $104.92 \pm 0.79$ & 0.88 \\
\hline Heart rate $[\mathrm{bpm}]$ & $68.78 \pm 4.47$ & $68.90 \pm 2.51$ & 0.47 \\
\hline IMT CA [mm] & $0.94 \pm 0.08$ & $0.92 \pm 0.08$ & 0.41 \\
\hline IMT CA bifurcation [mm] & $1.31 \pm 0.12$ & $1.29 \pm 0.14$ & 0.06 \\
\hline PWV CA [m/s] & $8.06 \pm 1.08$ & $7.51 \pm 0.79$ & 0.06 \\
\hline PWV AA [m/s] & $8.00 \pm 0.92$ & $7.55 \pm 1.06$ & 0.24 \\
\hline EDVD (\%) & $7.84 \pm 1.01$ & $8.02 \pm 0.77$ & 0.25 \\
\hline Total cholesterol [mmol/L] & $5.83 \pm 0.40$ & $5.75 \pm 0.36$ & 0.35 \\
\hline Triglycerides [mmol/L] & $1.77 \pm 0.33$ & $1.61 \pm 0.14$ & 0.07 \\
\hline LDL cholesterol [mmol/L] & $4.69 \pm 0.49$ & $4.77 \pm 0.40$ & 0.72 \\
\hline HDL cholesterol [mmol/L] & $1.03 \pm 0.10$ & $1.04 \pm 0.09$ & 0.09 \\
\hline Glucose [mmol/L] & $4.70 \pm 0.32$ & $4.61 \pm 0.26$ & 0.18 \\
\hline Insulin $[\mu \mathrm{U} / \mathrm{mL}]$ & $16.01 \pm 2.61$ & $16.80 \pm 3.03$ & 0.28 \\
\hline
\end{tabular}


Table 2. Comparison of patients with pseudo-resistant and true resistant arterial hypertension (AH)

\begin{tabular}{|c|c|c|c|}
\hline Indicators & $\begin{array}{l}\text { Pseudo-resistant AH } \\
\quad(\mathrm{n}=27)\end{array}$ & $\begin{array}{l}\text { True resistant AH } \\
\quad(\mathrm{n}=21)\end{array}$ & p \\
\hline HOMA-IR & $3.34 \pm 0.57$ & $3.44 \pm 0.64$ & 0.44 \\
\hline $\mathrm{HbA}_{1 \mathrm{c}}(\%)$ & $4.65 \pm 0.33$ & $4.71 \pm 0.27$ & 0.17 \\
\hline Total antioxidant activity [mmol/L] & $1.22 \pm 0.05$ & $1.16 \pm 0.05$ & 0.00 \\
\hline $\mathrm{MDA}[\mathrm{nmol} / \mathrm{mL}]$ & $32.24 \pm 1.53$ & $33.24 \pm 1.76$ & 0.01 \\
\hline $\mathrm{DC}[\mathrm{nmol} / \mathrm{mL}]$ & $32.54 \pm 2.78$ & $29.95 \pm 3.54$ & 0.03 \\
\hline IL-6 [ng/mL] & $109.55 \pm 7.44$ & $111.91 \pm 8.31$ & 0.06 \\
\hline $\mathrm{CRP}[\mathrm{ng} / \mathrm{mL}]$ & $4.32 \pm 0.61$ & $4.66 \pm 0.77$ & 0.17 \\
\hline Aldosteron [ng/dL] & $14.34 \pm 1.23$ & $15.09 \pm 1.31$ & 0.04 \\
\hline Renin [ng/mL/hour] & $2.57 \pm 0.44$ & $2.50 \pm 0.50$ & 0.86 \\
\hline ARR & $5.80 \pm 1.38$ & $6.35 \pm 1.64$ & 0.23 \\
\hline Adiponectin [ng/mL] & $7.44 \pm 0.64$ & $8.93 \pm 0.67$ & 0.00 \\
\hline Leptin [ng/mL] & $13.72 \pm 1.93$ & $11.46 \pm 1.34$ & 0.00 \\
\hline IVSd [sm] & $1.15 \pm 0.09$ & $1.16 \pm 0.13$ & 0.64 \\
\hline IVSs [sm] & $1.37 \pm 0.13$ & $1.34 \pm 0.14$ & 0.36 \\
\hline TPWd [sm] & $1.14 \pm 0.12$ & $1.15 \pm 0.17$ & 0.15 \\
\hline TPWs [sm] & $1.51 \pm 0.28$ & $1.60 \pm 0.44$ & 0.70 \\
\hline TIVSd [sm] & $5.01 \pm 0.35$ & $5.07 \pm 0.48$ & 0.78 \\
\hline TIVSs [sm] & $3.19 \pm 0.27$ & $3.22 \pm 0.34$ & 0.63 \\
\hline EF $(\%)$ & $65.82 \pm 1.82$ & $65.90 \pm 2.74$ & 0.33 \\
\hline LVM [g] & $262.76 \pm 57.30$ & $274.26 \pm 92.09$ & 0.45 \\
\hline LVMMI $\left[\mathrm{g} / \mathrm{m}^{2}\right]$ & $126.05 \pm 29.86$ & $132.08 \pm 42.89$ & 0.72 \\
\hline RWT & $0.46 \pm 0.04$ & $0.46 \pm 0.04$ & 0.91 \\
\hline LAD [mm] & $37.38 \pm 3.65$ & $37.19 \pm 3.47$ & 0.21 \\
\hline $\mathrm{AD}[\mathrm{mm}]$ & $31.98 \pm 0.68$ & $32.18 \pm 0.44$ & 0.01 \\
\hline Mean pulmonary AP [mm Hg] & $15.75 \pm 3.06$ & $15.64 \pm 2.93$ & 0.29 \\
\hline $\mathrm{RAV}[\mathrm{mL}]$ & $37.14 \pm 3.71$ & $37.73 \pm 4.00$ & 0.61 \\
\hline LAV [mL] & $48.42 \pm 4.46$ & $48.18 \pm 3.67$ & 0.80 \\
\hline $\mathrm{E}[\mathrm{cm} / \mathrm{s}]$ & $66.09 \pm 6.70$ & $69.28 \pm 5.04$ & 0.07 \\
\hline$A[\mathrm{~cm} / \mathrm{s}]$ & $75.83 \pm 7.33$ & $73.94 \pm 8.25$ & 0.26 \\
\hline$E / A$ & $0.89 \pm 0.12$ & $0.91 \pm 0.10$ & 0.05 \\
\hline DT $[s]$ & $0.16 \pm 0.12$ & $0.13 \pm 0.03$ & 0.11 \\
\hline IVRT [s] & $0.11 \pm 0.02$ & $0.12 \pm 0.03$ & 0.07 \\
\hline $\mathrm{E}[\mathrm{cm} / \mathrm{s}]$ & $11.33 \pm 2.03$ & $11.87 \pm 2.58$ & 0.47 \\
\hline $\mathrm{E} / \mathrm{e}$ & $6.00 \pm 1.15$ & $6.10 \pm 1.39$ & 0.86 \\
\hline
\end{tabular}

A - maximum late (atrial) filling speed; AA — aortic arch; AP — artery pressure; ARR — aldosterone-renin ratio; BMI — body mass index; $\mathrm{BP}$ - blood pressure; $\mathrm{CA}$ - carotid artery; CRP — C-reactive protein; $\mathrm{DBP}$ — diastolic blood pressure; $D C$ - diene conjugates; $D T$ - time of deceleration early diastolic flow rate; $E$ - filling rate in spectral mode; $e$ - maximum early LV filling rate at tissue mode; $E / A$ - ratio of maximal

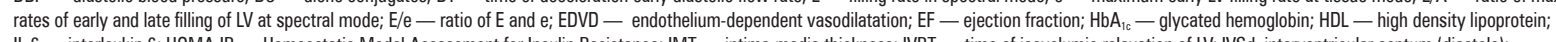
IL-6 - interleukin 6; HOMA-IR - Homeostatic Model Assessment for Insulin Resistance; IMT — intima-media thickness; IVRT — time of isovolumic relaxation of LV; IVSd, interventricular septum (diastole); IVSs, interventricular septum (systole); LDL — low-density lipoprotein; LVM — left ventricular mass; MDA — malonic dialdehyde; RAV — right atrial volume; LAV — left atrial volume; SBP — systolic blood pressure; PWV - pulse wave velocity; TIVSd - thickness of the interventricular septum (diastole); TIVSs - thickness of the interventricular septum (systole); TPWd - thickness of the posterior wall of the left ventricle in diastole; TIVSd — the thickness of the interventricular septum in diastole

is responsible for a wide range of side effects in the cardiovascular system, which, due to the increased concentration of angiotensin II and aldosterone, lead to an increase in BP, an increase in extracellular fluid volume, activation of hypercoagulation, and accel- eration of fibrosis processes in the heart and vessels [11-14].

It is known that most obese individuals have a high level of blood aldosterone, and as the obesity progresses, the concentration of this hormone 
increases. A number of studies have found a clear correlation between aldosterone levels and BMI. The results of a large Framingham Offspring Study show a direct relationship between elevated plasma aldosterone levels and high BP in 1688 patients [15]. It is possible that secondary hyperaldosteronism accompanying obesity also contributes greatly to the development of AH in these patients. This relationship is well observed in studies on the reduction of excess body weight, where weight loss entails a decrease in the concentration of aldosterone in the blood and is accompanied by a decrease in BP $[5,9,13]$.

Aldosterone has also been shown to adversely affect carbohydrate metabolism through three major mechanisms: IR, reduced insulin production, and activation of gluconeogenesis $[3,6,9,12]$.

The data obtained in our work show that patients with resistant hypertension differed from non-resistant patients with more pronounced cardiovascular remodeling, IR and metabolic disorders; it can be explained by the negative effect of aldosterone, which levels are significantly higher in $\mathrm{RH}$.

Significantly lower BMI in patients with true resistance could be explained by their sufficient physical activity, whereas pseudo-resistant patients in our study did not follow the physician's recommendations for providing adequate levels of physical activity. That is why, in the absence of a difference in BMI values between true resistant and pseudo-resistant patients at the beginning of the study, patients with true RH after complex 6-month therapy (including increased physical activity) had significantly lower BMI than pseudo-resistant ones. Higher adiponectin levels and lower leptin levels with true resistance can be explained by a large decrease in body weight of these patients compared to pseudo-resistant ones. It is known that in lower body weight, adiponectin is higher and leptin is lower than in higher body weight. The more pronounced imbalance of the system of oxidative stress-antioxidant protection in patients with true $\mathrm{RH}$ can be explained by significantly higher levels of aldosterone, compared to pseudoresistant patients.

\section{Limitations of the study}

Our study included only patients of a certain age group (45-55 years old). Perhaps in other age groups (or a wider age range) hemodynamic and metabolic parameters with true and pseudo-resistant $\mathrm{AH}$ would have different features. A limitation of our work is also that adherence to the therapy of our patients was evaluated according to their diaries, which may be somewhat subjective. In addition, when evaluating the effectiveness of antihypertensive therapy, we took into account the data of office and home BP monitoring, but did not perform ambulatory BP monitoring, which is a more sensitive method for identifying masked uncontrolled hypertension.

\section{Conclusions}

Thus, in our work it was found that patients with resistant hypertension differed from hypertensive obese patients without resistance by having higher BMI and BP, higher levels of triglycerides, insulin, $\mathrm{HbA1c}$, more pronounced IR, cardiovascular remodeling, imbalance of oxidative stress-antioxidant protection system, higher proinflammatory and RAAS activity. Patients with true resistance differed from pseudoresistant patients by having significantly lower BMI, higher aldosterone levels more pronounced imbalance of the system of oxidative stress-antioxidant protection and less pronounced adipokines imbalance.

\section{References}

1. Williams B, Mancia G, Spiering W, et al. 2018 ESC/ESH Guidelines for the management of arterial hypertension. Eur Heart J. 2018; 39(33): 3021-3104, doi: 10.1093/eurheartj/ehy339.

2. Chernova I, Krishnan N. Resistant Hypertension Updated Guidelines. Curr Cardiol Rep. 2019; 21(10): 117, doi: 10.1007/s11886019-1209-6, indexed in Pubmed: 31471727.

3. Gorini S, Marzolla V, Mammi C, et al. Mineralocorticoid Receptor and Aldosterone-Related Biomarkers of End-Organ Damage in Cardiometabolic Disease. Biomolecules. 2018; 8(3), doi: 10.3390/ biom8030096, indexed in Pubmed: 30231508.

4. Ezequiel DG, Paula RB, Lovisi JC, et al. [Spironolactone reduces blood pressure and albuminuria of obese hypertensive patients with metabolic syndrome]. J Bras Nefrol. 2013; 35(1): 69-72, doi: 10.5935/01012800.20130011, indexed in Pubmed: 23598755.

5. Hannich M, Wallaschofski H, Nauck M, et al. Physiological Aldosterone Concentrations Are Associated with Alterations of Lipid Metabolism: Observations from the General Population. Int J Endocrinol. 2018; 2018: 4128174, doi: 10.1155/2018/4128174, indexed in Pubmed: 29780416.

6. DuPont JJ, Jaffe IZ. 30 years of the mineralocorticoid receptor: The role of the mineralocorticoid receptor in the vasculature. J Endocrinol. 2017; 234(1): T67-T82, doi: 10.1530/JOE-17-0009, indexed in Pubmed: 28634267.

7. Wada T, Ishikawa A, Watanabe E, et al. Eplerenone prevented obesity-induced inflammasome activation and glucose intolerance. J Endocrinol. 2017; 235(3): 179-191, doi: 10.1530/JOE-17-0351, indexed in Pubmed: 28855315.

8. Min SeH, Kim SeH, Jeong InK, et al. Independent Association of Serum Aldosterone Level with Metabolic Syndrome and Insulin Resistance in Korean Adults. Korean Circ J. 2018; 48(3): 198-208, doi: 10.4070/kcj.2017.0200, indexed in Pubmed: 29557106.

9. Ramalingam L, Menikdiwela K, LeMieux M, et al. The renin angiotensin system, oxidative stress and mitochondrial function in obesity and insulin resistance. Biochim Biophys Acta Mol Basis Dis. 2017; 1863(5): 1106-1114, doi: 10.1016/j.bbadis.2016.07.019, indexed in Pubmed: 27497523.

10. Pizoń T, Rajzer M, Wojciechowska W, et al. The relationship between plasma renin activity and serum lipid profiles in patients with primary 
arterial hypertension. J Renin Angiotensin Aldosterone Syst. 2018; 19(4): 1470320318810022, doi: 10.1177/1470320318810022, indexed in Pubmed: 30404585.

11. Rossier BC, Staub O, Hummler E. Genetic dissection of sodium and potassium transport along the aldosterone-sensitive distal nephron: importance in the control of blood pressure and hypertension. FEBS Lett. 2013; 587(13): 1929-1941, doi: 10.1016/j. febslet.2013.05.013, indexed in Pubmed: 23684652.

12. Funder JW. Aldosterone and Mineralocorticoid Receptors-Physiology and Pathophysiology. Int J Mol Sci. 2017; 18(5), doi: 10.3390/ ijms18051032, indexed in Pubmed: 28492512.
13. Luther JM. Effects of aldosterone on insulin sensitivity and secretion. Steroids. 2014; 91: 54-60, doi: 10.1016/j.steroids.2014.08.016, indexed in Pubmed: 25194457.

14. Tesch GH, Young MJ. Mineralocorticoid Receptor Signaling as a Therapeutic Target for Renal and Cardiac Fibrosis. Front Pharmacol. 2017; 8: 313, doi: 10.3389/fphar.2017.00313, indexed in Pubmed: 28611666

15. Kathiresan S, Larson MG, Benjamin EJ, et al. Clinical and genetic correlates of serum aldosterone in the community: the Framingham Heart Study. Am J Hypertens. 2005; 18(5 Pt 1): 657-665, doi: 10.1016/j. amjhyper.2004.12.005, indexed in Pubmed: 15882548. 